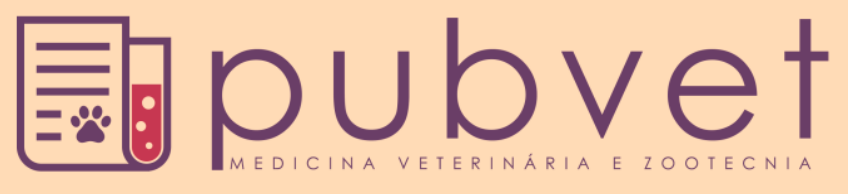

https://doi.org/10.22256/pubvet.v12n2a27.1-9

\title{
Desenvolvimento e zoometria de caprinos leiteiros jovens de diferentes grupos genéticos
}

\author{
Leandro Santos e Silva ${ }^{\bullet}$, Darlan Silva dos Santos ${ }^{\ominus 2}$, Edneide Rodrigues da Silva ${ }^{\ominus 2}$, \\ Jordânia Kely Barbosa da Silva ${ }^{\ominus}$, Gislaine Alexandrino da Silva ${ }^{\ominus 2}$, Gisele Maria Nunes \\ Vieira ${ }^{\ominus 2}$, Greicy Mitzi Bezerra Moreno ${ }^{3}$ \\ ${ }^{I}$ Mestrando(a) em Ciência Animal e Pastagens pela Universidade Federal Rural de Pernambuco, Unidade acadêmica de \\ Garanhuns. Garanhuns - PE Brasil. \\ ${ }^{2}$ Graduando(a) em Zootecnia pela Universidade Federal de Alagoas, Campus Arapiraca - AL Brasil. \\ ${ }^{3}$ Professora da Universidade Federal de Alagoas, Campus Arapiraca - AL Brasil. E-mail: greicymitzimoreno@yahoo.com.br \\ *Autor para correspondência, E-mail: ses.leandro@gmail.com
}

RESUMO. Objetivou-se com o presente trabalho avaliar o efeito do grupo genético sobre o desenvolvimento e zoometria de caprinos leiteiros jovens. O estudo foi desenvolvido no município de Coité do Nóia, AL, mensurando-se 166 cabritos Saanen, Toggenburg, Alpina Americana, Parda Alpina, Murciana e Mestiços. A partir do desmame (60 dias), e a cada 21 dias, até 144 dias de idade, foram realizadas as pesagens dos animais e as seguintes medidas zoométricas: comprimento corporal, perímetro torácico, altura da cernelha, altura da garupa, largura de peito, largura de garupa, comprimento de garupa e perímetro escrotal. Os resultados foram avaliados por analises de variância e as médias foram comparadas pelo teste de Tukey a $5 \%$ de probabilidade. $\mathrm{O}$ efeito da idade sobre a zoometria foi avaliado por análise de regressão. Não houve efeito $(P>0,05)$ para a interação entre grupo genético e idade. Em relação à influência do grupo genético sobre a zoometria, houve diferença, onde os grupos Parda Alpina e mestiços apresentaram médias superiores $(\mathrm{P}<0,01)$ aos demais grupos avaliados para comprimento corporal $(51,6 \mathrm{~cm}, 50,6 \mathrm{~cm})$, altura de garupa $(52,7$ $\mathrm{cm}, 52,5 \mathrm{~cm})$, largura de garupa $(10,0 \mathrm{~cm}, 10,1 \mathrm{~cm})$ e perímetro escrotal $(12,7 \mathrm{~cm} ; 13,3$ $\mathrm{cm})$. Para o gênero, as fêmeas apresentaram média superior aos machos apenas para comprimento corporal $(49,7 \mathrm{~cm})$. O grupo genético, o gênero e a idade são fatores que influenciam o desenvolvimento e a zoometria de caprinos leiteiros jovens.

Palavras chave: Caprinocultura, condições áridas, grupos exóticos, medidas biométricas

\section{Development and zoometry of young dairy goats from different genetic groups}

ABSTRACT. The objective of this work was to evaluate the effect of the genetic group on the development and zoometry of young dairy goats. The study was developed in the municipality of Coité do Nóia, AL. It was used measuring 166 goats kids Saanen, Toggenburg, Alpina Americana, Parda Alpina, Murciana and mixed breed animals (NDRP). After weaning (60 days), and every 21 days, up to 144 days of age, the animals were weighed and the following zoometric measures: body length, thoracic perimeter, height of the withers, height of the rump, breast width, croup width, croup length and scrotal perimeter. The results were evaluated through the analysis of variance and the mean was tested by the Tukey test at 5\% probability. The effect of age on the zoommetry was evaluated by regression analysis. There was no effect $(P>0.05)$ on the interaction between genetic group and age. In relation to the influence of the genetic group on the zoometry, there was a difference, in which the groups Parda Alpina and NDRP presented higher 
averages $(\mathrm{P}<0.01)$ to the other groups evaluated for body length $(51.6 \mathrm{~cm}, 50.6 \mathrm{~cm})$, croup height $(52.7 \mathrm{~cm}, 52.5 \mathrm{~cm})$, croup width $(10.0 \mathrm{~cm}, 10.1 \mathrm{~cm})$ and scrotal perimeter $(12.7 \mathrm{~cm}$, $13.3 \mathrm{~cm})$. For the genus, females presented a mean superior to males only for body length $(49.68 \mathrm{~cm})$. The genetic group, the genus and the age are factors that influence the development and the zoometry of young dairy goats.

Keywords: Arid conditions, biometric measurements, exotic groups, goat breeding

\section{Desarrollo y zoometría de caprinos lecheros jóvenes de diferentes grupos genéticos}

RESUMEN. Se objetivó con el presente trabajo evaluar el efecto del grupo genético sobre el desarrollo y zoometría de caprinos lecheros jóvenes. El estudio fue ejecutado en el municipio de Coité del Nóia, AL, midiendo 166 cabritos Saanen, Toggenburg, Alpina Americana, Parda Alpina, Murciana y mestizos. A partir del destete (60 días), y a cada 21 días, hasta 144 días de edad, se realizaron los pesajes de los animales y las siguientes medidas zoométricas: longitud corporal, perímetro torácico, altura de la cruz, altura de la grupa, anchura de pecho, anchura de grupa, longitud de la grupa y perímetro escrotal. Los resultados fueron evaluados a través de análisis de varianza y las medias fueron comparadas por el test de Tukey al 5\% de probabilidad. El efecto de la edad sobre la zoometría fue evaluado por análisis de regresión. No hubo efecto $(\mathrm{P}>0,05)$ para la interacción entre grupo genético y edad. En cuanto a la influencia del grupo genético sobre la zoometría, hubo diferencia, donde los grupos Parda Alpina y mestizos presentaron promedios superiores $(\mathrm{P}<0,01)$ a los demás grupos evaluados para longitud corporal $(51,6 \mathrm{~cm}, 50,6$ $\mathrm{cm})$, altura de grupa $(52,7 \mathrm{~cm}, 52,5 \mathrm{~cm})$, anchura de grupa $(10,0 \mathrm{~cm}, 10,1 \mathrm{~cm})$ y perímetro escrotal $(12,7 \mathrm{~cm}, 13,3 \mathrm{~cm})$. Para el género, las hembras presentaron media superior a los machos sólo para longitud corporal $(49,7 \mathrm{~cm})$. El grupo genético, el género y la edad son factores que influencian el desarrollo y la zoometría de los caprinos lecheros jóvenes.

Palabras clave: Caprinocultura, condiciones áridas grupos exóticos, medidas biométricas

\section{Introdução}

No Brasil, a caprinocultura leiteira é uma das alternativas indicadas para a geração de emprego e renda no campo, especialmente nos programas de fortalecimento da agropecuária familiar, favorecendo assim a diminuição do êxodo rural (Simplício et al., 2004, Dal Monte et al., 2010). O rebanho caprino nacional é estimado em 9,3 milhões de cabeças, dos quais $92,7 \%$ estão localizados na região Nordeste, que por sua vez, é responsável por mais de $70 \%$ da produção nacional de leite de cabra (ANUALPEC, 2017). No entanto, para otimizar esta produção são necessárias pesquisas que avaliem grupos genéticos adequados às condições nacionais, visando atender a demanda de mercado e otimizar a eficiência de produção.

O monitoramento da potencialidade dos animais é essencial para a elevação da produtividade, sendo que o acompanhamento do crescimento é de fundamental importância para o controle da produtividade do rebanho. Dessa forma, as medidas corporais e os índices zootécnicos são de fundamental importância para a caracterização de um grupo genético e conhecimento do seu potencial para exploração comercial. Assim, as informações obtidas permitem a comparação entre rebanhos de localidades diferentes e contribui para a definição de um padrão racial, servindo como referencial para programas de melhoramento genético (Valdez et al., 1981). É importante ressaltar que o grupo genético é uma subdivisão da espécie e na espécie caprina, dividem-se em troncos, dentre os quais o europeu e o africano são os que detêm a maioria dos grupos genéticos especializados na produção de leite (Ferreira et al., 2014). As diferenças raciais entre os troncos podem influenciar na eficiência de um grupo genético a uma determinada condição ambiental. Os grupos genéticos caprinos utilizados para produção de leite no Brasil, normalmente são grupos exóticos originários das regiões de clima temperado, criados puros ou em cruzamento com grupos nativos. No Nordeste, a maioria dos rebanhos caprinos é formada por animais considerados Mestiços ou "Crioulos", produto de cruzamentos 
indiscriminados entre grupos exóticos e nacionais. A conservação desses recursos genéticos locais representa uma grande contribuição para manutenção da diversidade genética dos animais, que são extremamente adaptados às condições edafoclimáticas da região. Entretanto, ainda são escassos os estudos avaliando o desenvolvimento e zoometria desses grupos genéticos nas diferentes regiões, microclimas e sistemas de produção do país.

Neste sentido, objetivou-se com este trabalho, avaliar o efeito do grupo genético sobre o desenvolvimento e zoometria de caprinos leiteiros jovens.

\section{Material e Métodos}

O trabalho foi realizado no município de Coité do Nóia (- $09^{\circ} 37^{\prime} 56^{\prime \prime}$ de latitude e $36^{\circ} 34^{\prime} 43^{\prime \prime}$ de longitude), Agreste Alagoano, no período de fevereiro a setembro do ano de 2014. Foram utilizadas informações de animais distribuídos em cinco diferentes grupos genéticos caprinos: Saanen, Parda Alpina, Alpina Americana, Murciana e Toggenburg. Além de informações de animais mestiços.

As matrizes do capril eram criadas em sistema de confinamento, agrupadas em baias coletivas com piso ripado, suspenso e acesso a solário. Permaneciam separadas dos reprodutores que se encontravam a uma distância de 50 metros das matrizes, criados em baias individuais em aprisco de piso ripado e suspenso.

A estação de cobrição dos animais ocorreu no período de fevereiro a maio do ano de 2014, com duração total de 70 dias. A detecção do cio foi realizada com auxílio de um macho rufião sempre no final da tarde, para incitar e detectar o cio nas matrizes. Uma vez confirmado o cio, a cabra era levada à baia do reprodutor, previamente escolhido e permanecia com ele até ser coberta. Após a cobrição, era confirmada a gestação das cabras pela não repetição do cio. Aquelas que repetiam cio retornavam ao lote em estação de cobrição. Caso as cabras repetissem o cio, eram dadas mais duas chances, desde que não ultrapassasse o tempo total de 70 dias, tempo este, limite da estação de cobrição. Após o período de cobrição, as cabras gestantes permaneceram em lotes separados das demais. A estação de nascimento ocorreu entre 23 de julho a 14 de setembro de 2014, período chuvoso na região.

Os cabritos nascidos vivos passaram pelos cuidados básicos, como corte e cura do umbigo e foram identificados por colares numerados e pesados. Os animais foram mantidos confinados, em baias coletivas juntos com as mães, onde os mesmos eram amamentados e recebiam a partir dos 20 dias de idade uma pequena quantidade de sólidos para o rápido desenvolvimento ruminal. A ração concentrada consistia do sistema de creep feeding com capim verde picado e água a vontade.

Aos 60 dias de idade, os cabritos foram desmamados e novamente pesados. Após o desmame, os animais foram vermifugados e mantidos em confinamento, onde permaneceram em baias separadas e classificados pelo sexo, sendo arraçoados à vontade 3 vezes ao dia com dieta formulada para atender suas exigências nutricionais (NRC, 2007). A dieta consistia em capim elefante (Pennisetum purpureum Schum.) picado e concentrado comercial para cabritos leiteiros, além de água à vontade.

A partir do desmame dos cabritos (60 dias) e com repetição a cada 21 dias até atingirem 144 dias de idade foram realizadas as medidas zoométricas, com auxílio de fitas métricas e compassos adaptados, de acordo com cada medida. Sendo assim, foram realizadas cinco mensurações: aos 60, 81, 102, 123 e 144 dias de idade. Nos mesmos dias das mensurações, a partir do desmame, os cabritos foram pesados, também totalizando cinco pesagens. As medidas zoométricas foram realizadas seguindo metodologias propostas por Salgueiro \& Nunes (1999) e, sendo elas: comprimento corporal (CC), perímetro torácico (PT), altura da cernelha (AC), altura da garupa (AG), largura do peito (LP), largura da garupa (LG), comprimento de garupa (CG), perímetro escrotal nos machos (PE) e escore corporal.

Foram registradas as medidas corporais de 166 cabritos dos grupos genéticos Saanen $(n=49)$, Parda Alpina $(\mathrm{n}=25)$, Murciana $(\mathrm{n}=9)$, Alpina Americana $(n=31)$, Toggenburg $(n=25)$ e mestiços $(\mathrm{n}=27)$ dos 60 aos 144 dias de idade, sendo 75 machos e 91 fêmeas. O delineamento utilizado foi o inteiramente casualizado, os resultados foram avaliados através de análises de variância e as médias foram comparadas pelo teste de Tukey a 5\% de probabilidade e análise de regressão pelo programa estatístico SAEG versão 9.1.

\section{Resultados e Discussão}

Os resultados apresentados na tabela 1 mostram que houve influência do grupo genético 
sobre a zoometria dos animais. Os grupos Parda Alpina e mestiços apresentaram médias superiores para as variáveis analisadas. Animais mestiços apresentam alta rusticidade e melhor desenvolvimento e adaptação às condições ambientais e ao nível tecnológico empregado nas criações, em comparação aos grupos genéticos exóticos (Ribeiro, 2004); sendo importante a interação entre animal e ambiente quando se busca melhor eficiência na exploração pecuária.

Tabela 1. Médias de zoometria em função do grupo genético de caprinos leiteiros jovens

\begin{tabular}{|c|c|c|c|c|c|c|c|c|}
\hline \multirow[b]{2}{*}{ Variável $^{1}$} & \multicolumn{6}{|c|}{ Grupo genético } & \multirow[b]{2}{*}{ Valor de $\mathrm{P}$} & \multirow[b]{2}{*}{$\mathrm{CV}(\%)$} \\
\hline & Mestiços & $\begin{array}{l}\text { Parda } \\
\text { Alpina } \\
\end{array}$ & $\begin{array}{c}\text { Alpina } \\
\text { Americana }\end{array}$ & Saanen & Toggenburg & Murciana & & \\
\hline $\mathrm{CC}(\mathrm{cm})$ & $50,60^{\mathrm{A}}$ & $51,62^{\mathrm{A}}$ & $47,52^{\mathrm{B}}$ & $49,11^{\mathrm{AB}}$ & $50,82^{\mathrm{A}}$ & $47,20^{\mathrm{B}}$ & $\mathrm{P}<0,01$ & 11,1 \\
\hline $\mathrm{PT}(\mathrm{cm})$ & $55,21^{\mathrm{A}}$ & $52,70^{\mathrm{B}}$ & $51,50^{\mathrm{B}}$ & $52,59^{\mathrm{A}}$ & $55,19^{\mathrm{A}}$ & $50,10^{\mathrm{B}}$ & $\mathrm{P}<0,01$ & 8,0 \\
\hline $\mathrm{AC}(\mathrm{cm})$ & $51,23^{\mathrm{AB}}$ & $51,52^{\mathrm{A}}$ & $48,32^{\mathrm{C}}$ & $49,65^{\mathrm{ABC}}$ & $50,89^{\mathrm{AB}}$ & $48,93^{\mathrm{BC}}$ & $\mathrm{P}<0,01$ & 7,7 \\
\hline $\mathrm{AG}(\mathrm{cm})$ & $52,54^{\mathrm{A}}$ & $52,68^{\mathrm{A}}$ & $49,57^{\mathrm{C}}$ & $50,70^{\mathrm{BC}}$ & $51,83^{\mathrm{AB}}$ & $49,93^{\mathrm{BC}}$ & $\mathrm{P}<0,01$ & 7,6 \\
\hline $\mathrm{LP}(\mathrm{cm})$ & $14,85^{\mathrm{A}}$ & $14,18^{\mathrm{ABC}}$ & $13,85^{\mathrm{BC}}$ & $13,78^{\mathrm{C}}$ & $14,68^{\mathrm{AB}}$ & $13,30^{\mathrm{C}}$ & $\mathrm{P}<0,01$ & 12,4 \\
\hline LG $(\mathrm{cm})$ & $10,11^{\mathrm{A}}$ & $10,02^{\mathrm{A}}$ & $8,94^{\mathrm{B}}$ & $9,52^{\mathrm{AB}}$ & $9,74^{\mathrm{A}}$ & $9,33^{\mathrm{AB}}$ & $\mathrm{P}<0,01$ & 14,6 \\
\hline $\mathrm{CG}(\mathrm{cm})$ & $16,70^{\mathrm{A}}$ & $16,65^{\mathrm{AB}}$ & $15,03^{\mathrm{D}}$ & $15,81^{\mathrm{BCD}}$ & $16,05^{\mathrm{ABC}}$ & $15,36^{\mathrm{CD}}$ & $\mathrm{P}<0,01$ & 11,6 \\
\hline $\mathrm{PE}(\mathrm{cm})$ & $13,31^{\mathrm{A}}$ & $12,68^{\mathrm{A}}$ & $10,30^{\mathrm{B}}$ & $12,80^{\mathrm{A}}$ & $13,33^{\mathrm{A}}$ & $10,90^{\mathrm{AB}}$ & $\mathrm{P}<0,01$ & 35,2 \\
\hline EC (1-5) & $3,48^{\mathrm{AB}}$ & $3,12^{\mathrm{BC}}$ & $3,03^{\mathrm{C}}$ & $3,01^{\mathrm{C}}$ & $3,67^{\mathrm{A}}$ & $3,40^{\mathrm{ABC}}$ & $\mathrm{P}<0,01$ & 22,3 \\
\hline
\end{tabular}

Médias seguidas por letras diferentes na mesma linha diferem entre si pelo teste de Tukey a 5\% de probabilidade. ${ }^{1}$ Variável: $\mathrm{CC}=$ comprimento corporal, $\mathrm{PT}=$ perímetro torácico, $\mathrm{AC}=$ altura da cernelha, $\mathrm{AG}=$ altura de garupa, $\mathrm{LP}=$ largura de peito, $\mathrm{LG}=$ largura de garupa íleos, $\mathrm{CG}=$ comprimento de garupa, $\mathrm{PE}=$ perímetro Escrotal, $\mathrm{EC}=$ escore corporal.

Houve influência da idade sobre a zoometria dos animais (Tabela 2), sendo esta fase dos 60 aos 144 dias a de maior desenvolvimento corporal dos caprinos jovens, conhecida como fase pré-púbere, em que sofrem uma maior influência do hormônio do crescimento ( $\mathrm{GH}$, somatotropina), que regula o crescimento somático e a diferenciação (Zachmann, 1992). Não houve efeito significativo para a interação entre grupo genético e idade, na zoometria de caprinos leiteiros jovens.

Tabela 2. Médias de zoometria em função da idade e interação entre grupo genético e idade de caprinos leiteiros jovens de diferentes grupos genéticos

\begin{tabular}{|c|c|c|c|c|c|c|c|c|c|}
\hline \multirow{2}{*}{ Variável $^{1}$} & \multicolumn{5}{|c|}{ Idade (dias) } & \multirow{2}{*}{ Equação $^{2}$} & \multirow{2}{*}{ Valor de $\mathrm{P}$} & \multirow{2}{*}{ Grupo genético $x$ Idade $^{3}$} & \multirow{2}{*}{$\mathrm{CV}(\%)$} \\
\hline & 60 & 81 & 102 & 123 & 144 & & & & \\
\hline $\mathrm{CC}(\mathrm{cm})$ & 45,42 & 47,42 & 49,65 & 52,32 & 52,82 & $\mathrm{Y}_{1}$ & $\mathrm{P}<0,01$ & 0,854 & 11,1 \\
\hline $\mathrm{PT}(\mathrm{cm})$ & 48,23 & 51,45 & 53,58 & 54,45 & 56,70 & $\mathrm{Y}_{2}$ & $\mathrm{P}<0,01$ & 0,999 & 8,0 \\
\hline $\mathrm{AC}(\mathrm{cm})$ & 46,08 & 47,98 & 51,02 & 52,16 & 53,22 & $\mathrm{Y}_{3}$ & $\mathrm{P}<0,01$ & 0,995 & 7,7 \\
\hline $\mathrm{AG}(\mathrm{cm})$ & 47,05 & 49,18 & 52,43 & 53,08 & 54,22 & $\mathrm{Y}_{4}$ & $\mathrm{P}<0,01$ & 0,992 & 7,6 \\
\hline $\mathrm{LP}(\mathrm{cm})$ & 13,25 & 13,46 & 14,49 & 14,58 & 14,75 & $\mathrm{Y}_{5}$ & $\mathrm{P}<0,01$ & 0,988 & 12,4 \\
\hline $\mathrm{LG}(\mathrm{cm})$ & 8,90 & 9,26 & 9,83 & 9,97 & 10,08 & $\mathrm{Y}_{6}$ & $\mathrm{P}<0,01$ & 0,762 & 14,6 \\
\hline $\mathrm{CG}(\mathrm{cm})$ & 14,74 & 15,21 & 16,45 & 16,46 & 16,80 & $\mathrm{Y}_{7}$ & $\mathrm{P}<0,01$ & 0,674 & 11,6 \\
\hline $\mathrm{PE}(\mathrm{cm})$ & 9,30 & 11,05 & 11,58 & 13,41 & 15,75 & $\mathrm{Y}_{8}$ & $\mathrm{P}<0,01$ & 0,999 & 35,2 \\
\hline EC (1-5) & 3,25 & 3,37 & 3,22 & 3,45 & 3,30 & $\mathrm{Y}_{9}=3,31$ & 0,979 & 0,996 & 22,3 \\
\hline
\end{tabular}

Para a medida de comprimento corporal (CC), houve influência do grupo genético, tendo sido os grupos genéticos Parda Alpina, Toggenburg e mestiços os que apresentaram as maiores médias $(\mathrm{P}<0,01)$ em comparação aos demais grupos, com valores de $51,6 \mathrm{~cm}, 50,8 \mathrm{~cm}$ e $50,6 \mathrm{~cm}$, respectivamente. No Nordeste, o grupo genético Parda Alpina é o mais representativo dentre os caprinos leiteiros, principalmente no semiárido, o que permitiu pela seleção natural, melhor adaptação as condições ambientais da região, sendo bastante utilizada em programas de cruzamento para produção de mestiços leiteiros (Simplício et al., 2004, Malheiros Filho et al., 2014). Possivelmente os animais mestiços do presente estudo apresentaram influência da 
genética alpina, permitindo um melhor desenvolvimento corporal, semelhante ao do grupo exótico.

Segundo Ribeiro (1997) o comprimento corporal (CC) está diretamente relacionado à produção leiteira, tornando-se um importante parâmetro de avaliação nos grupos genéticos selecionados para este fim. Pelo CC podem-se selecionar animais com melhor desempenho reprodutivo, formando um melhor rebanho leiteiro. Assim, quando se faz a seleção de animais é possível que as características desejáveis sejam transmitidas para seus descendentes e que esses venham a produzir de forma mais eficiente (Ferreira et al., 2014).

Menezes et al. (2012) trabalhando com medidas zoométricas de caprinos de 5 grupos genético distintos (Alpino, $1 / 2$ Boer $+1 / 2$ Alpino, $1 / 2$ Anglo Nubiano $+1 / 2$ Alpino, $3 / 4$ Boer $+1 / 4$ Alpino, $1 / 4$ Boer $+1 / 4$ Alpino $+1 / 2$ Anglo Nubiano (Tricross) e diferentes pesos ao abate, com idade média de 145 dias relataram que o grupo genético influenciou as características de CC, em que os caprinos do grupo Alpino apresentaram média de $69,5 \mathrm{~cm}$, resultado este superior ao encontrado neste estudo para o mesmo grupo $(51,6 \mathrm{~cm})$.

Para as medidas de perímetro torácico (PT), os grupos mestiços, Toggenburg e Saanen apresentaram as maiores médias, com valores de $55,2 \mathrm{~cm}, 55,2 \mathrm{~cm}$ e $52,6 \mathrm{~cm}$, respectivamente. Segundo Santana et al. (2001), a medida corporal de perímetro torácico, assim como comprimento de corpo, altura da cernelha e da garupa são importantes. Essas medidas podem indicar, por exemplo, a capacidade digestiva e respiratória dos animais, bem como, características produtivas como rendimento de carcaça. O grupo genético Parda Alpina foi o que apresentou maiores médias para as medidas de altura da cernelha (AC) e altura da garupa (AG) com valores de $51,5 \mathrm{~cm}$ e $52,7 \mathrm{~cm}$, respectivamente. $\mathrm{O}$ grupo de animais mestiços também apresentou média superior para AG $(52,5$ $\mathrm{cm})$ em comparação com os demais grupos genéticos, não diferindo significativamente da Parda Alpina.

Em estudo desenvolvido por Gomes et al. (2011) trabalhando com características de carcaça de caprinos de cinco grupos raciais (Alpino, $1 / 2$ Boer $+1 / 2$ Alpino, $1 / 2$ Anglo Nubiano + 1/2 Alpino, $3 / 4$ Boer + 1/4 Alpino e 1/2 Anglo Nubiano + 1/4Boer $+1 / 4$ Alpino) criados em confinamento, com abate sendo realizado com média de 110 dias, observaram diferenças para AC. Os animais do grupo genético Alpino apresentaram valor médio de 56,9 cm, superior aos encontrados neste trabalho.

De acordo com Ribeiro (1997) é desejável que as matrizes apresentem uma garupa longa, larga e ligeiramente inclinada, pois essas características estão diretamente relacionadas ao melhor desenvolvimento do feto e facilidade de parto, além de uma melhor implantação do úbere. $\mathrm{O}$ grupo genético Parda Alpina é considerado de grande porte, com peso adulto que pode chegar de 70 a $90 \mathrm{~kg}$ nas fêmeas, apresentando boa característica leiteira, o maior comprimento de garupa deste grupo pode estar relacionado a estas características citadas.

Para a medida de largura de peito (LP) o grupo de animais mestiços apresentou a maior média em comparação aos demais grupos genéticos, com valor de $14,8 \mathrm{~cm}$. Este resultado foi próximo ao resultado encontrado por Yáñez et al. (2004) em cabritos Saanen $(14,9 \mathrm{~cm})$ para esta largura. O grupo de mestiços também apresentou a maior média para largura de garupa (LG), assim como os grupos Parda Alpina e Toggenbung, com valores de $10,1 \mathrm{~cm}, 10,0 \mathrm{~cm}$ e $9,7 \mathrm{~cm}$, respectivamente. $O$ grupo genético Alpina Americana apresentou a garupa mais estreita, com valor de $8,9 \mathrm{~cm}$.

Para as medidas de comprimento de garupa (CG) e perímetro escrotal (PE), o grupo dos mestiços apresentou maiores valores, com $16,7 \mathrm{~cm}$ para CG e 13,3 cm para PE. Os grupos genéticos Toggenburg, Saanen e Parda Alpina também apresentaram as maiores médias para $\mathrm{PE}$, não diferindo $(\mathrm{P}<0,01)$ do grupo de mestiços. $\mathrm{O}$ grupo Alpina Americana apresentou menores médias para as duas variáveis. As medidas corporais e as pontuações atribuídas às características externas são consideradas de extrema importância. As mesmas desempenham o papel de auxiliar na escolha correta dos animais de acordo com o objetivo do produtor, demonstrando de forma direta a aptidão do grupo genético e podendo ser utilizadas para fornecer indicações para seleção de matrizes e reprodutores (Ferreira et al., 2014). No entanto, são poucos os trabalhos utilizando medidas morfométricas em caprinos leiteiros.

$\mathrm{O}$ perímetro escrotal $(\mathrm{PE})$ em animais jovens tem relação direta com o tamanho testicular, produção espermática, características físicas do sêmen e também com a fertilidade dos machos e das fêmeas aparentadas (meio-irmãs e filhas) (Peña et al., 2001). Neste estudo, o PE foi diferente 
apenas para o grupo genético Alpina Americana, com valor médio de $10,3 \mathrm{~cm}$.

O grupo genético Toggenburg apresentou maior escore corporal (EC) em relação aos demais grupos, com valor de 3,67 pontos. A avaliação do escore corporal é uma medida subjetiva baseada na classificação dos animais em função da massa de gordura e da cobertura muscular por meio de avaliação visual e/ou tátil que estima o estado nutricional (Machado et al., 2008).

Menezes et al. (2012) trabalhando com medidas biométricas de caprinos de cinco grupos raciais distintos (Alpino, $1 / 2$ Boer $+1 / 2$ Alpino, $1 / 2$ Anglo Nubiano + 1/2 Alpino, 3/4 Boer + 1/4 Alpino, $1 / 4$ Boer $+1 / 4$ Alpino $+1 / 2$ Anglo Nubiano (Tricross) e diferentes pesos ao abate, com idade média de 145 dias verificaram que o grupo racial influenciou o escore corporal (EC). Os caprinos do grupo genético Alpina apresentaram EC médio de 2,57, resultado este inferior ao obtido neste trabalho para o mesmo grupo $(3,12)$. Assim como Gomes et al. (2011) trabalhando com características de carcaça de caprinos de cinco grupos genéticos (Alpino, $1 / 2$ Boer + 1/2 Alpino, 1/2 Anglo Nubiano + 1/2 Alpino, 3/4 Boer + 1/4 Alpino, e $1 / 2$ Anglo-Nubiano $+1 / 4$ Boer $+1 / 4$ Alpino) criados em confinamento, com abate aos 110 dias, também puderam observar diferenças para EC, onde os animais do grupo Alpino também apresentaram valor médio menor de EC $(2,59)$ que os dois grupos com denominação Alpina avaliados neste estudo. Os animais do presente trabalho apresentaram média 3,3 para EC, indicando uma condição corporal boa de acordo com a classificação de (Cezar \& Sousa, 2006).

A influência do gênero sobre as medidas biométricas avaliadas está na figura 1.

Para o comprimento corporal houve diferença. As fêmeas apresentaram média superior aos machos, com valor de 49,7 cm. O comprimento corporal pode ser uma medida utilizada para predição do peso vivo, quando aliado a outras medidas, como o perímetro torácico, a altura da cernelha e da garupa e o comprimento de garupa. Wenceslau et al. (2000) apontam que existe uma correlação entre o comprimento corporal e a produção de leite. As fêmeas que apresentam maior CC tendem a ser mais produtivas. Desta forma, pode-se trabalhar a seleção de fêmeas leiteiras com maior $\mathrm{CC}$, a fim de incrementar a produção na propriedade. Fazer o acompanhamento destas medidas durante a fase de crescimento das cabritas é bastante importante, pois pode auxiliar na seleção das futuras matrizes ainda jovens.

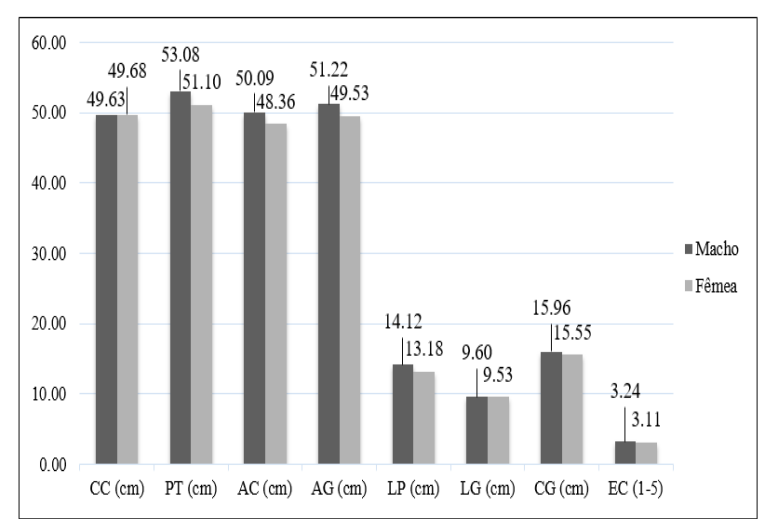

Figura 1. Medidas zoométricas em função do gênero de caprinos leiteiros jovens $\mathrm{CC}=$ Comprimento corporal; $\mathrm{PT}=$ Perímetro torácico $\mathrm{AC}=$ Altura de cemelha; $\mathrm{AG}=$ Altura de Garupa; LP = Largura de peito; LG Larura de garupa; CG Comprimento corporal; $\mathrm{EC}=$ Escore corporal

$\mathrm{O}$ perímetro torácico $(\mathrm{PT})$ foi maior nos machos em relação às fêmeas, com valores médios de $53,1 \mathrm{~cm}$ e $51,1 \mathrm{~cm}$, respectivamente, confirmando sua maior capacidade, por razões fisiológicas, de crescimento e desenvolvimento corporal. Silva et al. (2006) afirmam que há uma elevada correlação entre peso vivo e PT, sendo esta razão utilizada em barimetria. A barimetria é técnica pela qual se consegue estimar o peso vivo e outros aspectos corporais e produtivos, por meio de mensurações do corpo do animal vivo. Portanto, quanto maior o peso do animal, maior será o perímetro torácico.

Os machos apresentaram maiores médias para altura de cernelha (AC) e altura de garupa (AG) em relação às fêmeas, com valores de $50,1 \mathrm{~cm}$ e $51,2 \mathrm{~cm}$, respectivamente. No entanto, estas medidas não apresentaram interação entre gênero e idade. Oliveira et al. (2009) avaliando o desenvolvimento ponderal e a zoometria corporal de caprinos do grupo genético Anglonubiana criados em sistema semi-intensivo até 240 dias verificaram que a altura da cernelha foi, dentre as medidas, a que mais sofreu efeito do gênero. Até os 180 dias de idade foi superior dos machos de $6,5 \%$ em relação às fêmeas. Os mesmos autores verificaram que a influência do gênero sobre a medida de altura de garupa (AG) ocorreu até os 150 dias de idade com superioridade para os machos, assim como encontrado no presente trabalho até os 144 dias. Após esse período as médias não apresentaram diferença significativa.

As medidas de largura de peito (LP) bem como a largura de garupa (LG) foram diferentes entre 
machos e fêmeas, com médias de $14,1 \mathrm{~cm}, 9,6 \mathrm{~cm}$, $13,2 \mathrm{~cm}$ e $9,5 \mathrm{~cm}$, respectivamente. A largura de garupa é uma medida diretamente relacionada às características produtivas e reprodutivas. Fêmeas com garupas mais largas apresentam menos problemas de parto, bem como melhor implantação e desenvolvimento da glândula mamária, o que está diretamente relacionado com maior produção de leite e longevidade produtiva. Estes resultados discordam dos obtidos por Menezes et al. (2012), estudando o efeito do sexo de cinco grupos raciais distintos (Alpino, $1 / 2$ Boer $+1 / 2$ Alpino, $1 / 2$ Anglo Nubiano + $1 / 2$ Alpino, $3 / 4$ Boer $+1 / 4$ Alpino, $1 / 4$ Boer + 1/4 Alpino + 1/2 Anglo Nubiano (Tricross) sobre a zoometria de caprinos aos 25,30 e $35 \mathrm{~kg}$ relataram que o sexo influenciou algumas medidas. As fêmeas apresentaram maiores larguras de garupa (LG) e de peito (LP) em relação aos machos em todos os pesos ao abate avaliados. Segundo os mesmos autores, isto pode ter ocorrido em função da maior deposição de gordura subcutânea nas regiões dianteira e traseira do corpo das fêmeas.

O comprimento de garupa (CG) e escore corporal (EC) nos machos também foram maiores $(\mathrm{P}<0,01)$ em relação às fêmeas, com valores médios de 16,0 e $3,2 \mathrm{~cm}$, respectivamente. Os machos apresentam uma maior deposição muscular quando comparado com fêmeas, sendo a garupa, região anatômica que compõe o pernil, uma área de possível incremento muscular, com um notório desenvolvimento neste gênero. Menezes et al. (2012) avaliando o ganho de peso e medidas biométricas de caprinos jovens, encontraram médias para EC superiores para machos $(3,1)$ em relação às fêmeas $(2,9)$, assim como neste trabalho. No entanto, Gomes et al. (2011) ao estudarem a zoometria de caprinos de cinco grupos raciais (Alpino, $1 / 2$ Boer $+1 / 2$ Alpino, $1 / 2$ Anglo Nubiano + 1/2 Alpino, 3/4 Boer + 1/4 Alpino, e $1 / 2$ Anglo Nubiano $+1 / 4$ Boer $+1 / 4$ Alpino) criados em confinamento, observaram médias opostas para o EC, onde as fêmeas obtiveram maiores escores $(3,1)$ que os machos $(2,9)$. Um bom EC pode representar vantagem para o produtor, principalmente se os cabritos foram vendidos vivos para o abate, como é rotina em alguns criatórios.

Os pesos médios em função do gênero e idade dos cabritos leiteiros estão representados na figura $\underline{2}$.

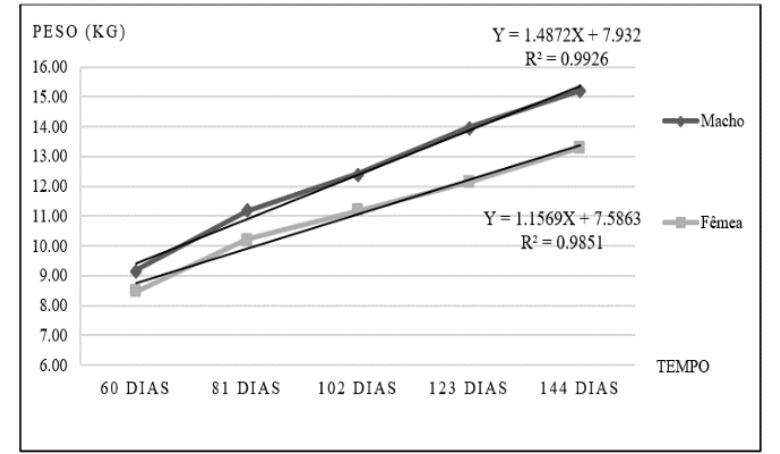

Figura 2. Peso médio de caprinos leiteiros jovens em função do gênero $\mathrm{x}$ idade

Houve influência do gênero nos pesos dos cabritos. Os machos foram mais pesados que as fêmeas nas cinco mensurações avaliadas. Os machos apresentaram pesos médios de 9,2; 11,2; 12,$4 ; 14,0$ e $15,2 \mathrm{~kg}$ e as fêmeas de 8,$5 ; 10,2 ; 11,2$; 12,2 e $13,3 \mathrm{~kg}$ para as pesagens aos $60,81,102$, 123 e 144 dias, respectivamente.

Alguns autores observaram a influência do sexo sobre os pesos de caprinos em diferentes idades, indicando geralmente superioridade dos machos em relação às fêmeas (Silva \& Araújo, 2000, Medeiros et al., 2004), em caprinos de vários grupos raciais. A superioridade dos pesos dos machos pode ser devido ao efeito anabólico dos hormônios sexuais secretados pelos fetos machos que, consequentemente são capazes de absorver mais nutrientes da mãe durante o desenvolvimento pré-natal (Medeiros et al., 2004).

\section{Conclusões}

O grupo genético e o gênero são fatores que influenciam o desenvolvimento e zoometria de caprinos leiteiros jovens sobre condições do Agreste Alagoano. A idade dos 60 até os 144 dias de idade influencia significativamente $o$ desenvolvimento e zoometria de caprinos jovens de aptidão leiteira, mantendo um crescimento linear durante essa fase.

\section{Referências Bibliográficas}

ANUALPEC. 2017. Anuário da Pecuária Brasileira, 20th edn. Instituto FNP, São Paulo, SP, Brasil.

Cezar, M. F. \& Sousa, W. H. 2006. Avaliação e utilização da condição corporal como ferramenta de melhoria da reprodução e produção de ovinos e caprinos de corte. Revista Brasileira de Zootecnia, 35, 649-678.

Dal Monte, H. L. B., Costa, R. G., Holanda Júnior, E. V., Pimenta Filho, E. C. \& Menezes, M. P. 
C. 2010. Mensuração dos custos e avaliação de rendas em sistemas de produção de leite caprino nos Cariris Paraibanos. Revista Brasileira de Zootecnia, 3, 2535-2544.

Ferreira, T. A., Pereira, I. G., Gouveia, A. M. G., Pires, A. V., Facó, O., Farah, M. M., Pessoa, M. C. \& Guimarães, M. 2014. Avaliação genética de caprinos da raça Saanen nascidos no Brasil de 1979 a 2009. Arquivo Brasileiro de Medicina Veterinária e Zootecnia, 66, 1179-1188.

Gomes, H. F. B., Menezes, J. J. L., Gonçalves, H. C., Cañizares, G. I. L., Medeiros, B. B. L., Polizel Neto, A., Lourençon, R. V. \& Chávari, A. C. T. 2011. Características de carcaça de caprinos de cinco grupos raciais criados em confinamento. Revista Brasileira de Zootecnia, 40, 411-417.

Machado, R., Corrêa, R. F., Barbosa, R. T. \& Bergamaschi, M. A. C. M. 2008. Escore da condição corporal e sua aplicação no manejo reprodutivo de ruminantes. Embrapa Pecuária Sudeste-Circular Técnica, 57, 1-16.

Malheiros Filho, J. R., Furtado, D. A., do Nascimento, J. W. B. \& Oliveira, C. J. B. 2014. Produção, qualidade do leite e índices fisiológicos de cabras Alpinas no semiárido no período chuvoso. Revista Brasileira de Engenharia Agricola e Ambiental-Agriambi, $18,762-768$.

Medeiros, L. F. D., Luna, M. C. M. d., Cabral Neto, O. \& Vieira, D. H. 2004. Avaliação de alguns aspectos de desempenho de caprinos da raça Anglo-nubiana, no Estado do Rio de Janeiro. Revista de Ciências da Vida, 24, 103118.

Menezes, J. J. L., Gonçalves, H. C., Cañizares, G. I. L., Rodrigues, L., Medeiros, B. B. L., Gomes, H. F. B., Marques, R. O. \& Emerson, M. S. 2012. Ganho de peso e medidas biométricas de caprinos jovens em função do gruporacial, peso de abate e sexo. Veterinária e Zootecnia. Veterinária e Zootecnia, 19, 574583.

NRC. 2007. Nutrient requirements of small ruminants: sheep, goats, cervids, and new world camelids, 7th rev. edn. Natl. Acad. Press, Washington, DC., Washington.

Oliveira, D. F., Cruz, J. F., Carneiro, P. L. S., Malhado, C. H. M., Rondina, D., Ferraz, R. d. C. N. \& Neto, M. R. T. 2009. Desenvolvimento ponderal e características de crescimento de caprinos da raça Anglonubiana criados em sistema semi-intensivo. Revista Brasileira de Saúde e Produção Animal, 10, 256-265.

Peña, C. D. O., Queiroz, S. A. \& Fries, L. A. 2001. Comparação entre critérios de seleção de precocidade sexual e a associação destes com características de crescimento em bovinos Nelore. Revista Brasileira de Zootecnia, 30, 93-100.

Ribeiro, F. L. 2004. A importância das cabras mestiças na produção de leite. O Berro, 64, 103-108.

Ribeiro, S. D. A. 1997. Caprinocultura: Criação Racional de Caprinos. Nobel, São Paulo.

Salgueiro, C. C. M. \& Nunes, J. F. 1999. Estudo de características testiculares e espermáticas de caprinos e ovinos. Revista Brasileira de Reprodução Animal, 23, 231-232.

Santana, A. F. S., Costa, G. B. \& Fonseca, L. S. 2001. Correlações entre peso e medidas corporais em ovinos jovens da raça Santa Inês. Revista Brasileira de Saúde e Produção Animal, 1, 74-77.

Silva, D. C., Azevêdo, D. M. M. R., Alves, A. A., Campelo, J. E. G., Oliveira, M. E. \& Malhado, C. H. M. 2006. Estimativa do peso vivo através do perímetro torácico de ovinos Santa Inês. Revista Científica de Produção Animal, 8, 4146.

Silva, F. L. R. \& Araújo, A. M. 2000. Desempenho produtivo em caprinos mestiços no Semi-árido do Nordeste do Brasil. Revista Brasileira de Zootecnia, 29, 1028-1035.

Simplício, A. A., Wander, A. E., Leite, E. R. \& Lopes, E. A. 2004. A caprino-ovinocultura de corte como alternativa para a geração de emprego e renda. In: EMBRAPA (ed.) Embrapa Caprinos. Documentos. EMBRAPA, Mossoró.

Valdez, C. A., Fagan, D. V. \& Vicera, I. B. 1981. The correlation of body weight to external body measurements in goats. Philippine Journal of Veterinary and Animal Sciences, 37, 62-89.

Wenceslau, A. A., Lopes, P. S., Teodoro, R. L., Verneque, R. S., Euclydes, R. F., Ferreira, W. \& Silva, M. A. 2000. Estimação de parâmetros genéticos de medidas de conformação, produção de leite e idade ao primeiro parto em vacas da raça Gir leiteiro. Revista Brasileira de Zootecnia, 29, 153-158.

Yáñez, E. A., Resende, K. T., Ferreira, A., Medeiros, A. N., Silva Sobrinho, A. G., Pereira 
Filho, J. M., Teixeira, I. A. M. A. \& Artoni, S. M. B. 2004. Utilização de medidas biométricas para predizer características da carcaça de cabritos Saanen. Revista Brasileira de Zootecnia, 33, 1564-1572.

Zachmann, M. 1992. Interrelations between growth hormone and sex hormones: physiology and therapeutic consequences. Hormone Research in Paediatrics, 38, 1-8.

\section{Article History:}

Received 10 October 2017

Accepted 27 October 2017

Available online 9 January 2018

License information: This is an open-access article distributed under the terms of the Creative Commons Attribution License 4.0, which permits unrestricted use, distribution, and reproduction in any medium, provided the original work is properly cited. 